\title{
Sociabilité sportive à Madagascar sous la colonisation française. La conquête d'un espace d'émancipation (1920-1939)
}

\section{Evelyne Combeau-Mari}

\section{Citer ce document / Cite this document :}

Combeau-Mari Evelyne. Sociabilité sportive à Madagascar sous la colonisation française. La conquête d'un espace d'émancipation (1920-1939). In: Outre-mers, tome 96, n³64-365, 2e semestre 2009. Le Sport dans l'Empire Français. Un instrument de domination coloniale ? pp. 91-106;

doi : https://doi.org/10.3406/outre.2009.4415

https://www.persee.fr/doc/outre_1631-0438_2009_num_96_364_4415

Fichier pdf généré le 26/04/2018 


\title{
Sociabilité sportive à Madagascar sous la colonisation française La conquête d'un espace d'émancipation (1920-1939)
}

Evelyne COMBEAU-MARI*

\begin{abstract}
A Madagascar, la vitalité du mouvement sportif associatif pendant la période de l'entre-deux-guerres interpelle. Il existe en effet une forme de contradiction entre le régime colonial, les interdits, la répression qu'il instaure et la prolifération de sociétés sportives qui encouragent les pratiques ludiques, le divertissement, la compétition. La vie associative sportive en contexte colonial assume une fonction essentielle de régulation sociale et politique. Son étude renseigne sur la nature des rapports de force qui se jouent entre colons et colonisés.

Dans les colonies françaises, le régime d'application du principe de liberté d'association défini par la loi de I90I est adaptable en fonction du contexte. Dans les "vieilles colonies ": Martinique, Guadeloupe, Guyane, Réunion ', acquises aux valeurs républicaines, la liberté d'association est promulguée sans restriction, si ce n'est quelques délais liés à l'acheminement des cablogrammes. La Réunion reçoit l'ordre d'application de la nouvelle législation enI908. Dans les colonies plus récentes, telles Madagascar, colonisée depuis $\mathrm{I} 896$, il n'est point question de liberté d'association, mais plutôt d'exercer un contrôle permanent afin d'éviter tout groupement susceptible de menacer l'ordre public. En matière de droit associatif, Madagascar comme de nombreux territoires est placée sous le régime de la législation antérieure, à savoir la loi du IO-I I avril I834 ${ }^{2}$ qui s'appuie essentiellement sur les dispositions de l'article 29I 3 du code pénal de I8ro. Cette législation prévaut jusqu'en I946.

Garantis contre les risques de subversion par cette législation drastique, gouverneurs généraux et administration coloniale mesurent au

* Université de La Réunion, CRESOI-EA 12.

I. Voir Combeau-Mari E., en collaboration avec Eve P., Fuma S., Linon J., Le mouvement associatif dans l'histoire de La Réunion, St Denis, Editions Graphica, 2002.

2. Ces textes sont rappelés dans leur intégralité dans un dossier du Gouvernement général de Madagascar. Côte CAOM-Gouvernement général de Madagascar : 6 (7) D67.

3. Code pénal-Section VII-Des associations ou réunions illicites.
\end{abstract}

Outre-Mers, T. 97, $N^{o}$ 364-365 (2009) 
lendemain de la Grande Guerre tout l'intérêt à promouvoir les exercices physiques en direction des populations colonisées à des fins patriotiques, hygiéniques, économiques et "civilisatrices 4 ". Tant que ces activités se cantonnent à la caserne et à l'école. Peu motivés par la préparation militaire et la gymnastique amorosienne, les Malgaches commencent à prendre goût aux pratiques sportives grâce à la dimension ludique des jeux de ballon : football et rugby. A compter des années vingt, les élites marquent un véritable engouement pour la vie associative qui se concrétise par le nombre et la vitalité des sociétés sportives. La possibilité d'un affrontement physique, voire d'une victoire sur le colonisateur explique pour part cet enthousiasme. Prenant conscience du phénomène, différents acteurs issus des milieux éducatifs laïcs, catholiques, ou protestants, s'emparent du sport pour attirer à eux cette jeunesse avide de jeux et de confrontations. Sa neutralité constitue un paravent commode à la poursuite d'objectifs plus directement idéologiques et politiques. A la fin des années trente, les sociétés sportives apparaissent aux élites malgaches comme un exceptionnel espace de liberté. L'association est encore le lieu privilégié d'apprentissage de l'organisation, de la responsabilisation et de l'émancipation.

\section{I - Une législation associative très scrupuleuse}

Les différents gouvernements ont toujours redouté dans l'association son caractère politique. Par ses modalités d'organisation et son unité d'opinion, elle incarne une forme de pouvoir ou de contre-pouvoir, qui peut menacer à terme le fonctionnement et même la légitimité de l'Etat 5. L'aboutissement législatif, symbolisé par la promulgation de la loi de I90I sous l'impulsion du président du Conseil WaldeckRousseau en France est l'exemple même d'une volonté politique portée par les Républicains soutenus par les Radicaux et Socialistes et d'un contexte favorable.

4. Les Européens se sentent investis de la mission de transmettre le "progrès " et sa philosophie dans les régions les plus reculées afin de sauver l'humanité. En France, la colonisation s'accompagne du principe d'assimilation des populations au modèle national. L'assimilation, destinée à transformer les indigènes en Français se définit comme "le processus par lequel un ensemble d'individus habituellement une minorité et/ou un groupe d'immigrants se fond dans un nouveau cadre social, plus large (...)"' (Encyclopédia Universalis, I998). Elle permet aux colonies de devenir des réservoirs de main d'œuvre et de soldats. Mais il faut aussi considérer le processus d'assimilation comme une volonté de transformation de la part de celui qui est assimilé dans une logique de promotion sociale, supposant l'adhésion aux valeurs du groupe dominant. Cette logique d'assimilation est indissociable de l'acculturation, processus par lequel un groupe assimile une culture différente de la sienne.

5. Jaume Lucien, "Une liberté en souffrance : l'association au XIx ${ }^{\text {e }}$ siècle" dans les Actes du colloque : "Les associations et le champ politique au $\mathrm{xx}^{\mathrm{e}}$ siècle", les $\mathrm{I} 6$ et I7 nov 2000 . 
“ Si le consensus existe sur la nécessité pour l'Etat républicain de reconnaître enfin la liberté fondamentale de s'associer, reste à savoir quelles garanties doivent lui permettre d'assurer sa prééminence face aux nouvelles entités sociales que représentent les associations " 6 .

Dans un contexte dominé par les troubles de l'affaire Dreyfus, le projet législatif sur les associations et son orientation anti-cléricale représentent un bon cheval de bataille pour maintenir et renforcer la cohésion de la majorité républicaine.

Dans le cadre colonial, la vigilance politique est renforcée. Ainsi, il n'est point question d'appliquer ce texte à Madagascar, occupée depuis I 896, mais plutôt d'exercer un contrôle permanent afin d'éviter tout groupement susceptible de menacer l'ordre colonial. L'étude des textes en vigueur dans la grande île permet déjà de percevoir l'atmosphère ambiante. En matière de droit associatif, Madagascar est placée sous le régime de la loi du IO-I I avril I $834^{7}$ qui s'appuie essentiellement sur les dispositions de l'article 29 I $^{8}$ du code pénal. L'article stipule :

"Art 29I - Nulle association de plus de 20 personnes dont le but sera de se réunir tous les jours ou à certains jours marqués pour s'occuper d'objets religieux, littéraires, politiques ou autres ne pourra se former qu'avec agrément du gouvernement et sous les conditions qu'il plaira à l'autorité publique d'imposer à la société. Dans le nombre de personnes indiquées par le présent article ne sont pas comprises celles domiciliées dans la maison où l'association se réunit.

L'autorisation donnée par le gouvernement est toujours révocable".

Il va de soi que toute dérogation à ces prescriptions s'accompagne de sanctions dissuasives précisées par les articles 292 et 294 du code pénal et renforcées par la loi du IO-II avril I834, notamment dans ses articles 2 et 3. A la dissolution immédiate de l'association, est ajoutée :

"Art 2 - Quiconque fait partie d'une association non autorisée sera puni de deux mois à un an d'emprisonnement de $50 \mathrm{~F}$ à $\mathrm{I} 000 \mathrm{~F}$ d'amende. En cas de récidive les peines pourront être portées au double. Le condamné pourra dans ce dernier cas être placé sous la surveillance de la haute police, pendant un temps qui n'excèdera pas le double du maximum de la peine. L'art $463 \mathrm{du}$ code pénal pourra être appliqué dans tous les cas.

Art 3 - seront considérés comme complices et punis comme tels ceux qui auront prêté ou loué sciemment leur maison ou appartement pour une ou plusieurs réunions d'une association non autorisée 9 ".

6. Lucas Nicolas, "L'adoption de la loi I901" dans les actes du colloque : "Les associations et le champ politique au $\mathrm{Xx}^{\mathrm{e}}$ siècle ", les $\mathrm{I} 6$ et $\mathrm{I} 7$ nov. 2000 .

7. Ces textes sont rappelés dans leur intégralité dans un dossier du Gouvernement général de Madagascar. Côte CAOM-Gouvernement génaral de Mada : 6 (7) D67.

Associations +réunions publiques-Législation à mada (une chemise).

8. Code pénal-Section VII-Des associations ou réunions illicites.

9. Référence à J.B. Duvergier, année I835, p. 59.

Outre-Mers, T. 97, $N^{\circ}$ 364-365 (2009) 
En réaction à l'affaire de la VVS ${ }^{\circ}$, première menace nationaliste tangible, qui agite l'opinion en pleine guerre mondiale (1915), le gouvernement général de Madagascar met en application le décret du 2 aôut 1848 sur les clubs :

"Art 13 - Les sociétés secrètes sont interdites. Ceux qui seront convaincus d'avoir fait partie d'une société secrète seront punis d'une amende de 100 à $500 \mathrm{~F}$, d'un emprisonnement de 6 mois à deux ans et de la privation des droits civiques de $\mathbf{I}$ an à 5 ans. Ces condamnations pourront être portées au double contre les chefs ou fondateurs des dites sociétés. Ces peines seront prononcées au préjudice de celles qui pourraient être encourues pour crime et délits prévus par les lois ${ }^{\mathrm{II}} \mathrm{M}$.

Considérant qu'il importe désormais d'astreindre "les associations ou sociétés indigènes autorisées " à une réglementation permettant de contrôler leur action, le gouverneur général Garbit opère un raidissement de la législation associative. La promulgation de l'arrêté "réglementant le fonctionnement des sociétés indigènes autorisées 12 " à Tananarive, le 19 janvier 1916 en témoigne :

"Art I - Aucune réunion, à quelque titre que ce soit d'une association ou société indigène autorisée ne pourra avoir lieu sans autorisation écrite du chef de province intéressée. Cette autorisation sera délivrée le cas échéant sur une demande formulée de trois membres du comité de la société, spécifiant l'objet exact de la réunion, l'heure et le lieu et précisant les questions qui doivent y être traitées.

Le chef de province aura la faculté, s'il le juge utile de déléguer un représentant de l'administration aux réunions".

Plus clairement sont visés les fonctionnaires et les élèves indigènes des écoles officielles et de formation des cadres qui avaient composé à l'image des élèves de l'école de médecine et de ceux de l'école Le-Myre-de-Vilers ${ }^{13}$ les forces vives du rassemblement de la VVS.

Io. VVS : Vy : fer, Vato: pierre, Sakelika : ramification; Société secrète sans statut, ni siège qui repose sur la cooptation de ces membres. Elle est considérée comme l'une des premières expressions du nationalisme malgache. Quelques personnalités, derrière des hommes d'église, caractérisées par un niveau plus élevé de formation s'engagent dans la voie de la réflexion politique, de l'écriture, du débat et participent de la prise de conscience nationale. Ses activités sont démasquées par l'administration coloniale avant toute forme d'intervention violente. Voir Ralaimihoatra Edouard, Histoire de Madagascar, Hachette édition Madagascar, Tananarive, 1969. p. 252.

II. Référence à J.B. Duvergier, année I 848 , p. 400. Décret du 25 mars-2 avril I 852 abroge celui du 28 juillet 1848 sur les clubs à l'exception de l'art $\mathrm{I} 3$. Le décret impérial du I 5 janvier-6 avril I 853 (portant application de diverses lois aux colonies) rend exécutoire le décret précédent aux colonies. Archives nationales de Madagascar(ANM) D I63-Souschemise "situation politique à Madagascar à la veille et pendant la guerre."

12. Côte CAOM-Gouvernement génaral de Madagascar: 6 (7) D67-Associations, réunions publiques-Législation à Madagascar.

13. L'école normale Le-Myre-de-Vilers forme les instituteurs malgaches. Créée dès l'année I 862 par les membres de la L.M.S, elle oriente les meilleurs élèves de l'enseignement primaire vers l'enseignement. Voir Koerner Francis, Histoire de l'enseignement privé et officiel à Madagascar 1820-1995, Paris, L'Harmattan, 1999, p. 4I. 
"Art 2 - Les fonctionnaires ou agents indigènes faisant partie d'un cadre régulier, les employés rétribués par l'administration, les auxiliaires, les élèves indigènes des écoles officielles ou des écoles spéciales ne pourront faire partie d'une association ou société indigène que sur autorisation générale du chef de la colonie, selon la société ou spéciale ou individuelle, délivrée par le chef de province s'il le juge opportun.

Art 3 - Toute infraction au présent arrêté entraînera la révocation ou le licenciement pour les fonctionnaires ou élèves des écoles ci-dessus spécifiées, sans préjudice des pénalités prévues par les lois et règlements et sera poursuivi envers les contrevenants, non compris dans ces deux catégories, conformément aux dispositions du code pénal ou à celles du code de l'indigénat.

Toute réunion d'indigènes-en dehors des associations ou sociétés autorisées, visées par le présent arrêté-reste soumise aux dispositions prévues par l'art I5 de l'arrêté du 4 décembreigr 2 réglementant le droit de répression par voie disciplinaire des infractions spéciales à l'indigénat ".

Outre l'aspect législatif, les circuits administratifs de création d'une association sont particulièrement contraignants. L'autorisation définitive est accordée par les services du gouvernement général de Madagascar et dépendances, traités par le secrétaire général ${ }^{14}$, après avis du procureur général, chef du service judiciaire de la colonie qui dans tous les cas demande contrôle aux services de la police. Les membres présumés de l'association donnent lieu à des rapports individuels de police ordonnés par le directeur des affaires politiques 15. C'est donc caractérisé par la mention "pas d'opposition d'ordre politique " que le dossier transite sans encombre au travers des rouages administratifs. Le formulaire d'autorisation de l'association est un arrêté du gouvernement général de Madagascar et dépendances qui demande sa mise en application au chef de région, sa publication au journal officiel, et son insertion au Gazetim-Panjakana (bulletin officiel en malgache). Toute modification des statuts de la dite association doit recevoir l'approbation préalable du gouverneur général. Malgré des conditions législatives et administratives décourageantes plus particulièrement pour les Malgaches, l'activité des associations sportives se révèle intense.

\section{II- Préparation physique et militaire : une politique d'assimila- tion pour les Malgaches}

Au lendemain de la Première Guerre mondiale, tout se passe dans la colonie comme si le pouvoir militaire prend l'ascendant auquel il ne peut plus prétendre en métropole. Cette domination se fixe plus énergiquement dans le domaine de l'éducation physique et des sports, avec

14. Les articles des associations sont épluchés et re-précisés si nécessaires selon la législation et les pratiques en vigueur.

15. La plupart des dossiers relatifs aux associations classés dans les dépôts d'archives portent la mention " confidentiel".

Outre-Mers, T. 97, No 364-365 (2009) 
pour ambition principale la préparation des jeunes Malgaches au service militaire et le désir d'un ancrage solide et irréversible à la nation française. Forte de 4I 000 engagés ${ }^{16}$ volontaires sur le front, la population malgache a démontré sa participation à l'effort de guerre. Il convient désormais de préparer l'avenir.

L'étude de la société Pro Patria, seconde société gymnique née à Madagascar est significative. Conçu à Tamatave, ce club est redevable à M. Grall, diplômé de l'école de Joinville alors sous-officier d'infanterie coloniale et professeur d'éducation physique au lycée Leconte de Lisle à St Denis de La Réunion. M. Grall est bien connu à La Réunion. Il est déjà à l'origine de la première société de gymnastique déclarée le 6 juillet I907, dénommée initialement $L$ 'Espérance et qui a pour but de préparer physiquement et militairement la jeunesse à la revanche. Fondée quasi-exclusivement sur la pratique de la gymnastique : agrès, mouvements d'ensemble, pyramides, cette société s'engage à "établir entre les jeunes gens des liens de fraternelle amitié, de fortifier en eux des sentiments de mutuelle solidarité et de développer le physique des élèves gymnastes. 17 " L'association, initialement ouverte dans le cadre du lycée, organise des exhibitions, des défilés et participe aux diverses cérémonies publiques : dont le I4 juillet. Elle étend ses activités au domaine des sports et à la société civile et prend le nom de Pro patria le I7 juin I9II. Comme de nombreuses sociétés de gymnastique de France métropolitaine, sa dénomination exprime les considérations patriotiques et nationalistes qu'elle promeut au travers de la pratique physique, à la veille du premier conflit mondial.

C'est donc son meilleur élève, Léon Vigoureux, diplômé et fraîchement sorti du même lycée de La Réunion avec tout un bagage concernant les sports qui poursuit son action sur la grande île. Arrivés tous dcux à Tamatave en plein conflit mondial, en juillet 1915, ils forment la société sportive et la dénomment sans originalité Pro Patria ${ }^{18}$. L'itinéraire de ce club illustre assez clairement l'évolution du dispositif associatif à Madagascar. Au delà de la préparation militaire, la société diffuse d'abord les sports individuels : athlétisme, natation, escrime, tir auprès des colons français, puis les sports collectifs tels le footballassociation et le rugby. La société prête son concours à diverses fêtes nationales comme "les journées de Galliéni ", les fêtes du I4 juillet, ainsi qu'aux réjouissances données à l'occasion du relèvement du franc et celles des contributions volontaires. Au service de l'œuvre patriotique et coloniale, le créole Léon Vigoureux est nommé pour une longue période moniteur d'éducation physique dans divers établissements scolaires de Tamatave, ce qui lui permet d'élargir son recrutement. Au lendemain du conflit, en manque de forces vives, la société ouvre ses

16. Ralaimihoatra Edouard, op. cit., p. 245.

17. fascicule célébrant le cinquantenaire de la Patriote édité aux imprimeries Cazal, La Réunion, 1970.

18. Madagascar Sports et cinéma, $\mathrm{n}^{\circ} 5$ du jeudi 2 mars I938-CAOM-POM $936 \mathrm{C}$ 
portes à la jeunesse tamatavienne, autorisant l'accès aux Malgaches. Elle modifie ses statuts et change de nom pour devenir la "FrancoMalgache 19 ".

"Article premier

Il est constitué à Tamatave sous le bienveillant patronage de Monsieur l'Administrateur-Maire une société dite "Sport Franco-malgache " laquelle a pour but de travailler à l'éducation physique et morale de la jeunesse par la pratique de la gymnastique, des sports et des réjouissances en plein-air ".

Par évidente obligation de neutralité, l'article I9 précise que "les discussions politiques ou religieuses sont expressément interdites." Tout manquement à cet article peut entraîner la radiation. Modèle du genre, son bureau prévoit en sus des incontournables président, viceprésident et trésorier : un "directeur sportif et quatre commissaires moniteurs ". L'accès des membres malgaches reste soumis à un filtre sérieux.

"Pour être admis comme membre actif il faut être présenté par deux parrains et être agréé par le conseil d'Administration qui statuera, s'il y a lieu par vote secret (...)".

De plus, "le comité doit être composé au moins de $2 / 3$ des membres européens ou assimilés."

Ouverte à Majunga quelques années plus tard, le $\mathrm{I}^{\mathrm{er}}$ décembre 1924, l'Union Sportive de Majunga, initiée par le lieutenant Naylis, de l'infanterie coloniale, en constitue un second exemple :

" cette société a pour but de travailler à l'éducation physique et morale de la jeunesse par la pratique des sports en général et des réjouisssances en plein-air ${ }^{20} "$.

Cautionné par l'avocat Couteau à la présidence et $M$. Vigne, Directeur du CNEP à la vice-présidence son "comité sportif " est composé de personnalités malgaches déjà bien insérées dans le tissu associatif : Rabé André, instituteur à la présidence, Rakotobe Pascal ${ }^{2 x}$, viceprésident, Robinson G, Trésorier, Ratovo Olivier et Rakotobe J.M., Commissaires. Manager, le lieutenant Naylis organise les séances d'éducation physique et enseigne les sports : gymnastique, footballassociation, cyclisme. Il accompagne le déroulement des fêtes officielles.

"Pendant que le Lieutenant s'occupait à inculquer à la jeunesse la technique de l'éducation physique, Rabe André essaya de son côté d'implanter le

19. Par arrêté du gouvernement général du 5 juillet 1917.

20. Madagascar Sports et cinéma, $\mathrm{n}^{\circ}$ Io du jeudi 7 avrili 938-CAOM-POM $936 \mathrm{C}$.

21. Pascal Rakotobe joue un rôle majeur dans le mouvement sportif entre-deux guerres. Il devient Manager du grand club malgache du stade Olympique de l'Emyrne. Il est élu dans les instances fédérales. 
football dans la vie des Majungais. Nos amateurs de tam-tam pour les Comoriens, de hira gasy ${ }^{22}$ pour les Merina, de moraingy ${ }^{23}$ pour les Sakalava et de rija ${ }^{24}$ pour les Betsiléo, étaient au début des anti-sportifs. Rabe engagea la bataille, se mit en contact avec le public $25 "$.

Le déroulement bi-annuel de grandes fêtes sportives patronnées par l'Union conquit progressivement le public. Avec la jeunesse catholique, à compter de I93I, de nouvelles sociétés sportives apparaissent dans l'agglomération majungaise : L'Olympique, Boanamary Athlétic Européen fondé par $M$. Walwein, devenu le président de la ligue régionale de la côte ouest.

Dans cette configuration, dynamisées par la circulaire Barthou du 22 mars $1920^{26}$, les associations gymniques et sportives se multiplient. Au delà de l'émergence de multiples sociétés de tir 27 : parrainées par les militaires telles que la société sportive et de tir de Majunga, le I 5.07.I905, la société de tir de Nosy-be, le 20.12.1924, la société de tir de Madagascar en 1926, la société de tir de Mananjary, le 05.01.1929., la société de tir de Tuléar en I931..., les clubs omnisports s'implantent dans la capitale et en province. A la fin des années vingt toutes les grandes et moyennes agglomérations sont dotées d'un et dans la grande majorité, de plusieurs clubs: Antsirabe et Majunga ${ }^{28}$ dès $1909^{29}$, Nosy-Be en I9I I 30 , Diégo-Suarez ${ }^{31}$ et Tamatave ${ }^{32}$ en I913, Vatomandry 33 en I9I4, Boanamary 34 en I918, Moramanga 35 et Mananjary 36 en 1923, Ankazobe 37, Brickaville 38, Fort-Dauphin 39, Farafangana 40 en I924, Tuléar ${ }^{41}$ en $1927 \ldots .$. Les bourgs les plus isolés en brousse possèdent leur cercle sportif. Les sociétés sportives quadrillent le territoire.

La ville de Tananarive affiche bien sûr une effervescence associative encore plus notable. Remarquons la création du Stade olympique de l'Emyrne, le I6 décembre I9I I, premier club tananarivien à ouvrir ses

22. Danse traditionnelle des Hauts-plateaux

23. Boxe traditionnelle

24. Pratique corporelle régionale

25. Madagascar Sports et cinéma, $\mathrm{n}^{\circ}$ ro du jeudi 7 avrilr938-CAOM-POM $936 \mathrm{C}$.

26. La circulaire Barthou du 22 mars 1920 vise à développer la préparation militaire dans le cadre de l'éducation physique scolaire.

27. Fournal officiel de Madagascar et dépendances. (FOMD).

28. Société du vélo-club majungais, 27.I I.1909. FOMD.

29. Union sportive d'Antsirabe, 15.04.1909, FOMD.

30. Association sportive de Nosy-Be, 07.0I.I9I I. FOMD.

31. Société sportive et récréative des jeunes gens 07.06.191 I. FOMD.

32. Seconde société créée : "l'Union sportive tamatavienne", le 29.ir.I913. FOMD.

33. Société sportive de Vatomandry, 19.12.1914. FOMD.

34. The Boanamary club, I918. FOMD.

35. Sport club de Madagascar, 22.12.1923. FOMD.

36. Stella club de Mananajary, 27.10.1923. FOMD.

37. Le malgache olympique d'Ankazobe, 26.04.1924. FOMD.

38. Cercle de Brickaville, 17.05.1924. FOMD.

39. The boanamary athlétic club, 21.06.1924. FOMD.

40. Le club farafanganais, 09.08.1924. fOMD.

41. Sport club de Tuléar, 18.06.1927. FOMD. 
portes aux élites malgaches. Emblématique, le stade constitue la référence aux yeux des autres clubs de la capitale tels le Iarivo Université Club né le 12 décembre 1913, la Jeunesse sportive tananarivienne d'Ambondrona créée le 06.09.1919., le Club printanier d'Isotry en date du 22.12.1923. Bientôt, tous les quartiers de Tananarive, y compris les plus populaires à l'image d'Isotry, sont identifiés à leur club sportif. Face à cet engouement, les Européens ouvrent le Racing club ${ }^{42}$ de Tananarive 18.12.1920, qui, comme son nom le rappelle, doit incarner à son plus haut niveau la représentation européenne.

Tananarive voit également la diversification des activités sportives et l'apparition des premiers clubs spécialisés, le plus souvent réservés aux colons européens. Sont ainsi ouverts successivement l'Automobile Club de Madagascar 43, le Club Hippique de Madagascar 44, le Tennis club tananarivien 45 , le Club Nautique de Tananarive ${ }^{46}$, ou bien le Golfing-club de Madagascar 47.

A compter des années vingt, de nouveaux promoteurs prennent conscience de l'attrait des pratiques sportives pour la jeunesse. Les missions catholiques et protestantes y voient un bon moyen pour ramener les jeunes à l'église. Ils initient les premières amicales des anciens 48 et ouvrent des clubs sportifs. Le collège jésuite St Michel de Tananarive déclare l'association St Michel alors que le Lycée laïc Gallieni lance son Union sportive lycéenne en I92I. Les protestants répliquent par la création du foyer chrétien le I0.05.1924. Les militaires renforcent leur position en créant des clubs spécifiques: Olympique Gallieni 49 et Sport militaire so en 1924. Les premiers mouvements de jeunesse s'organisent. Les Scouts de France sont fondés le 09.06.1923. Les Eclaireurs unionistes voient le jour le 03.01.1925.

Face à la croissance rapide et spectaculaire des effectifs malgaches dans certaines sociétés, le gouverneur général Marcel Olivier SI réitère en I925 la volonté ferme de greffer aux sociétés sportives une "préparation militaire". Dans son esprit, la formation physique des "Indigènes " doit reposer sur des finalités conscriptives et patriotiques avant tout. Il engage avec autorité un vaste recensement des associations sportives afin de vérifier l'existence ou bien d'implanter une "préparation militaire".

42. Le Racing club de France est un des premiers clubs créé à Paris en I 882 par l'élite étudiante bourgeoise. Il installe ses locaux dans le bois de Boulogne.

43. 27.01.1917. FOMD.

44. $07.02 .1925 \mathcal{F O M D .}$

45. 14.03.1925. FOMD.

46. 19.02.1927. FOMD.

47. 29.04.1930. FOMD.

48. Association amicale des professeurs, surveillants et anciens élèves des Frères des écoles chrétiennes de Tananarive, 12.06.1920; Association amicale des anciens élèves des Frères des écoles chrétiennes de Fianarantsoa, 27.I1.1920. FOMD.

49. 23.08.1924. FOMD.

50. 02.02.1924. FOMD.

5I. Archives Nationales de Madagascar-D I30.

Outre-Mers, T. 97, $N^{\circ} 364-365$ (2009) 
Signe du temps d'un assouplissement du régime colonial, cette proposition est accueillie de manière très aléatoire en fonction des lieux, de l'organisation et de l'historique des associations. Les structures qui entretiennent en 1925 des rapports encore très étroits avec les militaires demeurent évidemment les plus enclines à poursuivre ces objectifs.

Ainsi de la Société sportive de Diégo-Suarez, formée de 99 membres, dont 85 Européens et 14 asiatiques qui consacre l'essentiel de son activité à l'éducation physique des scolaires et qui souhaite depuis 1923 compléter cet enseignement physique par une éducation au tir. La réponse 52 apportée par le trésorier de la société le capitaine Merigeault à $M$. L'administrateur en chef des colonies, chef de la province de Diégo, datée du 22 novembre 1925 souligne cette permanence :

"Il serait à mon avis possible de créer une section de préparation militaire. La société en avait fait la demande en 1923 par une demande d'agrégation par le ministère de la guerre qui est restée en suspens. Dans cette section pourraient être admis les jeunes Créoles ou Malgaches en âge de suivre cette préparation. Un officier de la garnison aidé par un ou deux sous-officiers désignés par le colonel pourraient être chargés de la direction de cette instruction. La société n'hésiterait pas à fournir tous les moyens nécessaires. L'autorité militaire aurait à remettre le matériel, les munitions et la disposition du champ de tir pour l'instruction au tir".

Concrètement, pour développer la préparation militaire, il faut disposer d'un encadrement européen suffisant en nombre et en compétence. Cette double exigence n'est en définitive remplie que par un nombre infime de sociétés. L'exemple du Racing Ambondrona, plus gros club de la capitale vient le confirmer. Présidée par M. Borrel 53, la société dénombre 325 inscrits dont 125 Européens et 200 Indigènes. Pourtant présente dans les statuts, la section de préparation militaire n'a jamais pu être mise en place. Il faut bien reconnaître qu'en dehors de ces deux associations, les réponses apportées au gouverneur sous des motifs divers sont à considérer comme négatives.

Cette enquête administrative confirme au regard du nombre élevé de clubs l'engouement suscité par la vie associative et par le sport à Madagascar, toutes populations confondues. De préparation militaire, il n'est plus réellement question en 1925. Les pratiques physiques se concentrent autour des sports collectifs de grand terrain : association et rugby dans les clubs à fort effectif malgache. Alors que les Européens s'adonnent à des activités "distinctives": natation, tennis, golf... dans des structures fermées. Les associations qui émanent des établissements scolaires restent très attachées à l'athlétisme, comme école de

52. Lettre de la société sportive de Diégo envoyée par le trésorier de la société le capitaine Merigeault à $M$. L'administrateur en chef des colonies, chef de la province de Diégo, datée du 22 novembre 1925. ANM-Di30.

53. Courrier de M. Le Président du club Racing Ambondrona du 28 novembre 1925 à M. Le Président de la FSSAM. ANM Drzo. 
formation et de rigueur. Dans la promotion des pratiques sportives, les militaires sont désormais concurrencés par différents protagonistes issus des missions religieuses, des établissements scolaires laïcs et de la société civile.

\section{III- Le sport instrument de conquête de la liberté associative pour les Malgaches}

Il convient dès lors de s'interroger sur les effets d'une législation associative drastique. Interdits de groupements, de rassemblements, de réunions publiques 54 , les Malgaches ont trouvé fortuitement dans les sociétés de gymnastique, puis de sport un espace de liberté : espace de préparation et de confrontation physique d'abord, d'affirmation politique ensuite.

La capitale s'érige en siège privilégié de l'effervescence associative derrière un club emblématique le Stade olympique de l'Emyrne. Première émanation historique du sport malgache le club est créé par M. Paul Paoli 55, industriel et commerçant, fils du fameux Jean Paoli, pionnier du sport dans la colonie à la toute fin du $\mathrm{xIx}^{\mathrm{e}}$ siècle. A la différence du Sport-club, réservé exclusivement aux Européens, le stade compte 180 indigènes et 2 Européens en $1925^{56}$. A contrario de la gymnastique, le sport rencontre par sa dimension ludique l'adhésion des populations. Sur les Hauts-plateaux, il s'identifie quasi-exclusivement au rugby. Les clubs fleurissent dans les différents quartiers de la ville. Agréé par arrêté du I2 décembre 1913, le "Iarivo 57 sport Hova 58 " comprend en 1925128 membres malgaches et ne compte qu'un membre européen, le Président d'honneur : $M$. Lassalle, président de la Cour d'appel. Déclarée le 06.09.1919, la jeunesse sportive tananarivienne d'Ambandrona relève de l'initiative des commerçants d'Analakely 59 . Alors que le club européen du Racing ${ }^{60}$, formé essentiellement des élèves du lycée Gallieni subit les premières menaces face à l'adversité malgache, les dirigeants imaginent de rassembler les deux groupes. Le club évolue en Racing-Ambandrona par fusion le 25.04.1925.

54. Note du 23 novembre 1937 du Procureur général à $M$. le Gouverneur général de Madagascar portant sur les réunions. ANM Dizo.

55. Madagascar Sports et cinéma $n^{\circ}$ 6-Jeudi 10 mars 1938.

56. Courrier du Président du Stade olympique de l'Emyrne à $M$. le Président de la FSSAM de décembre r925. ANM, Dizo.

57. Iarivo est l'appellation stylisée d'Antananarivo.

58. Le club s'appelle depuis le 17 avril 1924 le "Nouvel Iarivo Sport Hova ", NISH. Courrier de 26 nov 1925 du Président du "Nouvel Iarivo Sport Hova " (N.I.S.H) à M. Le Président de la fédération des sociétés de Sport athlétique de Madagascar, ANM Dizo.

59. Du quartier marchand des pavillons d'Analakely en plein centre de Tananarive. Le club est présidé par le Dr Dartigolles. ANM, Dizo.

60. Fondé le 18.12.1920 et présidé par M. Lafosse. ANM D I30. 
"La jeunesse tananarivienne d'Ambandrona était perçue comme moins représentative de la nation malgache. Pour certains, elle était tout simplement un instrument utilisé par les colonisateurs pour briser le Stade olympique de l'Emyrne ${ }^{6 x}$ ".

Ses effectifs relativement équilibrés dans leur répartition : I25 Européens et 200 Malgaches lui confèrent un fonctionnement plus conforme aux objectifs d'assimilation prônés par l'administration. Ses résultats élogieux en championnat confortent sa représentativité. Le club remporte en 1925 les championnats de rugby (équipes $\mathrm{I}^{\text {ère }}$ et $2^{\mathrm{e}}$ ), de football, de cyclisme et d'athlétisme.

Un nouveau concurrent se révèle avec le "Iarivo Université Club 62 ", déclaré au Journal officiel en I923. Les quartiers les plus populaires de Tananarive ne sont pas en reste avec la création le 22.12.1923 du club printanier d'Isotry. Autre candidat au jeu collectif de combat, le Stellaclub ${ }^{63}$, inauguré en 1925 comporte 98 Malgaches et 2 européens parmi lesquels le Président d'honneur M. Lauseig, comptable des chemins de fer de Tananarive. L'engouement associatif ne se limite pas à la capitale et gagne du terrain en province.

Tamatave, centre des infrastructures portuaires et des échanges avec l'extérieur, bénéficie de la précocité du mouvement sportif. Très vite, la société dénommée "Sport franco-malgache " qui comprend 52 membres en 1925 soient 47 Européens, parmi lesquels on dénombre une majorité de créoles en provenance de La Réunion 64 et cinq membres indiens se voit concurrencée par la création de la société dite "Olympic club de la côte est-Tamatave-Madagascar " fondée le ro juin I923 65. La société présente des statuts conformes aux attentes administratives, mais affiche une toute autre répartition. La société admet 63 membres dont 3 Européens pour 60 Malgaches. Les trois Européens dont M. Rossary ${ }^{66}$, le directeur sportif assurent la délégation auprès du Comité sportif central de Tamatave et auprès de l'administration. Ils jouent en quelque sorte le rôle de caution morale. Le président, $M$. Jean Rajaona, écrivain, interprète, est une personnalité formée au collège St Michel déjà très active dans le milieu associatif tananarivien. Les autres membres malgaches du comité sont tous originaires de la bourgeoisie Merina, du corps des fonctionnaires.

6I. Entretien avec M. Ramanantsoa Todisoa, Président de la ligue de rugby d'Antananarivo le I9 février 2003 par Randriamaro Jean-Roland, "L'expression du politique par le populaire : l'exemple du rugby à Madagascar. ", Revue historique de l'océan Indien $n^{\circ} I$, Dynamiques dans et entre les îles du sud-ouest de l'océan indien ( $X V I I^{e}-X X^{e}$ siècle), 2005, p. 314 .

62. Présidé par M. Riddell. ANM Dizo.

63. Courrier du Président de la société sportive "Stella club" du 27 nov 1925 à M. Le Président de la FSSAM. ANM Dizo.

64. La communauté réunionnaise est venue renforcer la colonisation française particulièrement dans la région de Tamatave.

65. A.N.M. Dossier D I 30.

66. M. Rossary est à l'origine de la naissance de la société de gymnastique de Tananarive et impliqué dans les instances fédérales. 
Au milieu des années vingt, les Malgaches de la capitale et des Hauts-plateaux issus principalement des castes privilégiées ont investi massivement le mouvement sportif créé de toutes pièces par les Européens. Retenant les sports collectifs, comme moyen de combat symbolique, ils détournent les objectifs d'assimilation fixés par le colonisateur. Les matches témoignent physiquement de l'affrontement colonial. Le spectacle atteint des paroxysmes de ferveur populaire. Dans les faits, l'administration coloniale s'avère totalement débordée par une adhésion qu'elle pensait contenir. La lecture a posteriori des dossiers de constitution de certaines associations sportives qui éveillent l'attention des services administratifs révèle leur teneur éminemment politique.

Imprégnés de préjugés raciaux à l'égard des populations des Hautsplateaux, les administrateurs exercent déjà une surveillance assidue des sociétés gymniques et sportives avant l'affaire de la VVS. Cette méfiance se porte très nettement sur les groupements formés par les Malgaches de "race hova 67 ", réputés "rusés et frondeurs 68 ". Les descriptions établies dans les différents rapports coloniaux forcent le trait :

"Bien que plus faibles de corps, les Hovas ont une intelligence plus déliée ; ils se sont donnés une organisation politique; ils pratiquent avec plus de succès les travaux agricoles et les métiers manuels. Leur armée bien disciplinée avait fini par leur assurer la prééminence dans la plus grande partie de l'île. (....) On les représente ordinairement comme faux et fourbes ; ce défaut est en effet celui des courtisans de la haute-classe, fins diplomates, habitués à louvoyer entre toutes les difficultés pour maintenir leur crédit et perdre leurs adversaires 69 ".

L'importance accordée à des incidents somme toute bénins par certains chefs de province imbus de leurs prérogatives est significative de cette atmosphère. En juillet 1913, la Mutuelle sportive Hova de Diégo-Suarez, société en cours de formation décide pour renflouer sa trésorerie d'organiser un bal et invite un certain nombre de personnalités françaises et malgaches du district, mais informe en dernier lieu et tardivement le chef de province. La dénomination même de la société, rappelant sa représentativité "hova " est perçue par les administrateurs français comme une première provocation.

"Il y a quelques jours-le Io juillet au matin - j'appris par la rumeur publique que les Hovas devaient donner un bal le 12 dans l'ancien hôtel terminus. Renseignements pris il était exact qu'un comité avait lancé des invitations

67. L'administration française généralise à tort le terme "hova " pour parler des populations issues des Hauts-plateaux, les Merina. En fait, dans la langue malgache ce terme qualifie le second groupe statutaire dans la hiérarchie sociale de l'ancien régime : les roturiers (Hova) par opposition aux nobles (Andriana) ou aux esclaves (Andevo).

68. A.N.M. Dossier D 131 .

69. Vast Henri, La plus grande France, Bilan de la France coloniale, Paris, Garnier, 1909, p. 313. 
aux fonctionnaires européens-sauf au chef du district et à moi-avait fait une démarche près de $M$. Le Général Montignault pour qu'il assistât à cette fête, avait même demandé à l'agent - envoyer des drapeaux pour décorer la salle....tout cela sans m'en aviser et sans demander l'autorisation prévue par le paragraphe 15 de l'arrêté sur l'indigénat 70 ».

Blessé dans son amour-propre, le chef de province accorde l'autorisation mais dresse un réquisitoire très négatif de la communauté "Hova " de Diégo, qui selon lui trouve chez les Européens de nombreux appuis.

"Ces faits rapprochés de la lutte actuellement soutenue par les SaintMariens, montre un état d'esprit dont nous devons tenir compte pour l'avenir. Je n'entends pas étendre de plano cette opinion pessimiste aux Hovas de l'Imérina et j'espère qu'il ne s'agit que du petit groupe de Diégo. Notre ville où tant d'éléments se choquent, où toutes les races du monde sont représentées est une bien mauvaise école pour le maintien chez nos sujets malgaches de la déférence envers l'administration....et nous serons bientôt obligés de nous montrer plus sévères, si nous voulons maintenir l'ordre public".

En marge du texte au crayon de papier est apposée la mention :

"La société sportive Hova ne sera pas autorisée-Se montrer sévère vis à vis de tout indigène Hova ou autre qui manque de déférence vis à vis des représentants de l'administration \%.

La décision est confirmée par courrier du 30 juillet I9I3 du gouverneur général au chef de province. D’apparences anodines, ces frictions entre communautés ponctuent le quotidien et révèlent de plus profonds antagonismes. Les réticences à la formation de clubs en province sont presque toujours dictées par la suspicion de risques de subversion orchestrée par les groupements merina. Le fonctionnement de l'Union sportive d'Antsirabe ${ }^{71}$, autorisée depuis l'arrêté du I 2 février 1929, est remis en cause en 1935 au motif de l'effet d'entraînement et de propagande (nationaliste) des membres fondateurs. L'ouverture du " TCE de Moramanga 72 " est soumis à enquête préalable de ses adhérents :

"J'ai l'honneur de vous adresser ci-joint en communication un dossier composé de Io pièces relatif à une demande d'autorisation d'une association sportive dite "Club du TCE de Moramanga". Les écrivains comptables Ratsaralafy et Ramamonjisoa, membres du comité provisoire de la dite

70. Courrier de l'administrateur en chef des colonies-chef de la province à $M$. Le Gouverneur Général de Madagascar, Direction des affaires civiles (Confidentiel), le I2 juillet I9I3.ANM-D I3I-00006 Dossier concernant la Société sportive malgache de Diégo-suarez (1913).

71. ANM-D 13I- 00004 , Notice de renseignement relatif à l'Union sportive d'Antsirabé (U.S.A.), I 935 .

72. Un courrier confidentiel du 27 mars 1937 du Gouverneur Général de MadagascarBureau politique à l'administrateur supérieur chef de la région de Tananarive. CAOM, Gouvernement général de Madagascar : cote 6 (7) D67. 
société ont fait l'objet en 1935 de renseignements les représentant comme des individus très suspects au point de vue loyalisme. Afin de me permettre de prendre une décision en toute connaissance de cause, je vous prie de bien vouloir faire procéder à une enquête discrète sur l'attitude politique de ces fonctionnaires et m'en transmettre les résultats, résultats avec votre avis motivé et vos propositions tant sur les intéressés que sur le projet d'association lui-même ".

A compter des années trente, les notices de renseignements généraux s'intéressent de plus en plus aux sociétés sportives et témoignent de la surveillance accrue des participants. La "jeunesse sportive tananarivienne d'Ambondrona " attire plus particulièrement la vigilance depuis qu'elle a renouvelé son bureau pour l'année I937. L'élection comme vice-président de $M$. Pruvost et comme secrétaire de P. Dussac 73, principal propagandiste du Parti communiste français sur la Grande île surprend les services de police qui commentent :

"Le résultat inattendu de cette élection serait dû à une propagande politique faite auprès des joueurs 74 ".

La composition du bureau de L'union sportive des syndicats ouvriers et employés de Madagascar 75, suite à l'assemblée générale du 25 janvier 1939, ne prête pas à interprétation. Le club est présidé par M. Albertini 76 et désigne comme médecin Ravoahangy, amnistié de la VVS, et rallié au communisme. Elu député pour le MDRM en I946, il sera l'un des leaders de l'insurrection de 1947. Le club adopte la couleur rouge. Le discours d'Albertini et les échanges avec les sociétaires sont suffisamment explicites.

"Il faut, dit-il, avoir conscience de l'esprit sportif. Il ne faut pas être trop gourmand surtout au début. Il ne faut pas former non seulement des jeunes gens, mais aussi des jeunes-filles-Il faut amener vos filles et vos garçons car le sport ne fait pas distinction. Il faut former les enfants au sport non pour une future guerre, mais pour avoir plus tard des hommes solides. La Nation a besoin d'hommes solides. Il faut provoquer de nombreuses adhésions. Je ne crois pas que vous manquiez de moniteurs. Vous en trouverez deux ou trois au sein de votre assemblée (...) je regrette beaucoup que le camarade Ravoahangy ne soit pas présent aujourd'hui (...)mais il assistera assurément à la prochaine séance (....)".

73. Né en Russie à Simferopol en I896, Il est, avec Planque, le fondateur de la section malgache du parti communiste. Durant le mandat de Léon Cayla, il est incarcéré à plusieurs reprises. Il doit quitter Madagascar pour la France où il meurt à la fin de l'année 1937. Spacensky Alain, Madagascar, cinquante ans de vie politique, de Railamongo à Tsiranana, Paris, Nouvelles éditions latines, 1970, p. 29.

74. Extrait de rapports d'indicateurs du 7 décembre 1936. CAOM, Gouvernement général de Madagascar : cote 6 (7) D67.

75. Rapport des renseignements généraux du 28 janvier 1939. CAOM, Gouvernement général de Madagascar : cote 6 (7) D67.

76. M. Albertini est avocat à Tananarive et acquis aux idées communistes. Spacensky Alain, Madagascar, cinquante ans de vie politique, de Railamongo à Tsiranana, Paris, Nouvelles éditions latines, 1970 , p. 29.

Outre-Mers, T. 97, $N^{\circ} 364-365$ (2009) 
Lors de cette réunion, Rafaralahy André, restaurateur à Antanimena répond en ces termes à $M$. Albertini.

"Nous vous remercions Maître et Président. Nous voyons maintenant que feu Dussac a ressuscité en votre personne. Dussac mort, vous tenez sa place auprès de nous pour faire vivre cette société (...)".

Le milieu sportif, réservoir des élites malgaches apparaît à la veille de la Seconde Guerre mondiale largement infiltré par les groupements nationalistes. Aux objectifs sportifs se sont superposés des engagements idéologiques et politiques.

Malgré la pesanteur et les exigences de la législation, la sociabilité sportive s'épanouit pleinement à Madagascar pcndant la période de l'entre-deux-guerres. Cette vitalité remarquable sur la grande île trahit les nouvelles exigences de débat et de liberté auxquelles aspirent les populations malgaches citadines et instruites. Les militaires, pionniers du sport à Madagascar voient leur emprise décroître au profit des civils. Mais d'autres acteurs en prise avec la jeunesse investissent dans le modèle éducatif du sport. Fortes de leur antériorité et des formations dispensées auprès des populations locales, les missions protestantes d'abord, puis catholiques entendent également se positionner dans la partition du pouvoir sportif. Enthousiasmées par la dimension ludique et fédératrice de la sociabilité sportive, les élites des Hauts-plateaux s'emparent de cet espace potentiel de liberté et d'expression et le détournent au profit de visées politiques. L'association assume une fonction de formation des cadres nécessaire dans le combat d'émancipation nationale. L'entrée en guerre interrompt brutalement ces considérations associatives. 